\title{
METACYCLIC $p$-GROUPS AND THEIR CONJUGACY CLASSES OF SUBGROUPS
}

\author{
by ROLF BRANDL and LIBERO VERARDI $\dagger$
}

(Received 23 April, 1992)

Introduction. Let $G$ be a group and let $\mathscr{C}(G)$ be the set of all conjugacy classes $[H]$ of subgroups $H$ of $G$, where a partial order $\leq$ is defined by $\left[H_{1}\right] \leq\left[H_{2}\right]$ if and only if $H_{1}$ is contained in some conjugate of $\mathrm{H}_{2}$.

A number of papers (see for example [1] and the references mentioned there) deal with the question of characterizing groups $G$ by the poset $\mathscr{C}(G)$. For example, in [1] it was shown that if $\mathscr{C}(G)$ and $\mathscr{C}(H)$ are order-isomorphic and $G$ is a noncyclic $p$-group then $|G|=|H|$. Moreover, if $G$ is abelian, then $G \cong H$, and if $G$ is metacyclic then $H$ is metacyclic.

In this paper we improve upon the latter result. Indeed we have:

THEOREM. Let $G$ and $H$ be finite groups and assume that $\mathscr{C}(G)$ and $\mathscr{C}(H)$ are order-isomorphic. If $H$ is a noncyclic, metacyclic p-group, then $G \cong H$.

Clearly, the above result is not true if $H$ is cyclic. If $G$ is assumed to be finite, then $G$ is cyclic of prime power order, but the primes occurring may be different. Also, for infinite $G$, it does not seem to be clear, whether there is an analogue to the theorem, even in the case where $H \cong \mathbb{Z}_{4}$.

To prove the theorem we note first that, by the above, $G$ is a metacyclic $p$-group having the same order as $H$. The presentations of metacyclic $p$-groups given by $\mathrm{B}$. W. King [5] will be used frequently in the sequel. Indeed, for the remainder of this paper, let $G \cong\left\langle a, b \mid a^{p^{m}}=1, b^{p^{n}}=a^{p^{m-s}}, a^{b}=a^{\varepsilon+p^{m-r}}\right\rangle$, and similarly for $H$ with parameters $m^{\prime}, n^{\prime}$, $s^{\prime}, c^{\prime}, \varepsilon^{\prime}$. These parameters satisfy various arithmetic conditions, which can be found in [5, Theorem 3.2] (see also Lemma 2.1 below). In particular, $\varepsilon, \varepsilon^{\prime}= \pm 1$, and we speak about groups of type + if $\varepsilon=+1$ and of type - if $\varepsilon=-1$. Also, the notion of ordinary and exceptional metacyclic groups will be used (see [5, p. 110]). Moreover, if $(C, \leq)$ is a poset and $a, b \in C, a \leq b$, then $[a, b]=\{x \in C \mid a \leq x \leq b\}$ denotes the closed interval with end-points $a, b$.

All groups in this paper are finite.

1. The derived subgroup. In this section, we prove that the derived subgroup $G^{\prime}$ and the structure of $G / G^{\prime}$ can be read off from $\mathscr{C}(G)$ in the case where $G$ is a metacyclic $p$-group. Indeed, the following shows that normal subgroups with noncyclic factor group are determined by the structure of certain intervals in $\mathscr{C}(G)$.

Lemma 1.1. Let $G$ be a metacyclic $p$-group and let $R \leq G$. Assume that the interval $[[R],[G]]$ of $\mathscr{C}(G)$ is order-isomorphic to $\mathscr{C}(X)$, where $X$ is a noncyclic, abelian $p$-group. Then $R$ is normal in $G$ and $G / R \cong X$.

Proof. Proceed by induction on $[G: R]=|X|$. If $[G: R]=p^{2}$, then $[[R],[G]]$ is isomorphic to the modular lattice $\mu_{p+1}$. An inspection of $\left[N_{G}(R)\right]$ shows $R \varangle G$. So, assume $[G: R] \geq p^{3}$. Then there exists $Y \leq X,|Y|=p$, such that $X / Y$ is noncyclic. If [S]

† Work supported by M.U.R.S.T. of Itay.

Glasgow Math. J. 35 (1993) 339-344. 
corresponds to $Y$ via the assumed order-isomorphism, then by induction, we have $S \triangleleft G$ and $G / S \cong X / Y$ is abelian (and clearly metacyclic). Then $\Omega_{1}(X / Y) \cong \mathbb{Z}_{p} \times \mathbb{Z}_{p}$, and we see that $G$ contains a subgroup $T$ such that the interval $[[R],[T]]$ is isomorphic to $\mathcal{M}_{\mathrm{p}+1}$. Since $G / S$ is abelian, $T$ is normal in $G$. Moreover, $T$ contains (at least) $p+1$ conjugacy classes $\left[M_{1}\right], \ldots,\left[M_{p+1}\right]$ of maximal subgroups. Now, $G$ is metacyclic and so $T$ is 2 -generated. Thus, $M_{1}, \ldots, M_{p+1}$ are all the maximal subgroups of $T$ and all of them are normal in $G$ (note that $T \triangleleft G$ ).

Now $R \leq M_{1}^{x_{1}} \cap M_{2}^{x_{2}}$ for some $x_{1}, x_{2} \in G$ and by the above, we have $R \leq M_{1} \cap M_{2}=$ $\Phi(T)$, and $[T: R]=p^{2}$ implies $R=\Phi(T)$. Hence $R \triangleleft G$, as claimed.

The following consequence of Lemma 1.1 will be crucial for the proof of our main theorem.

Proposition 1.2. Let $G$ and $H$ be metacyclic p-groups and assume that $\varphi: \mathscr{C}(G) \rightarrow$ $\mathscr{C}(H)$ is an order-isomorphism. Then $\varphi\left(\left[G^{\prime}\right]\right)=\left[H^{\prime}\right]$. In particular, $G / G^{\prime} \cong H / H^{\prime}$ and $G^{\prime} \cong H^{\prime}$.

Proof. The first part follows from Lemma 1.1. Finally, $G^{\prime}$ and $H^{\prime}$ are cyclic groups of the same order and the result is proved.

At this point, we pause and discuss the question whether or not Proposition 1.2 could be true for arbitrary $p$-groups. The reader who may be interested in metacyclic $p$-groups only, is advised to continue with Section 2. Indeed, for the remainder of this section, let $G$ and $H$ be finite $p$-groups and assume that $\varphi: \mathscr{C}(G) \rightarrow \mathscr{C}(H)$ is an isomorphism of posets. Clearly, $\mathscr{C}(G)$ contains the lattice $\mathcal{N}(G)$ of all normal subgroups of $G$, and we conjecture that $\varphi(\mathcal{N}(G))=\mathcal{N}(H)$, that is $\varphi$ maps normal subgroups of $G$ onto normal subgroups of $H$. Indeed Proposition 3.4 below and the subsequent remark provide an affirmative answer in a special case. The conjecture would easily imply that, if $G$ is of maximal class or thin (see [2]), then $H$ has the analogous property. We do not have a counterexample to this, but the following shows that $G$ and $H$ may be of different nilpotency class and $\varphi([Z(G)]) \neq[Z(H)]$.

EXAMPLE 1.3. Let $G=\left\langle x, y, z \mid x^{9}=y^{9}=z^{9}=[x, z]=[y, z]=1,[x, y]=z\right\rangle$ and $H=\left\langle a, b, c \mid a^{9}=b^{9}=c^{9}=[b, c]=1,[a, b]=c,[a, c]=c^{3}\right\rangle$. Then $G$ and $H$ have order $3^{6}, G$ is of class 2 and $H$ is of class 3 and [3] implies that $\mathcal{N}(G)$ is order-isomorphic to $\mathcal{N}(H)$. Moreover, using "CAYLEY" and some straightforward, if tedious, computations done by hand, one can show that there is an order-isomorphism $\varphi$ from $\mathscr{C}(G)$ onto $\mathscr{C}(H)$. Here, we have $\varphi\left(\left[G^{\prime}\right]\right)=\left[H^{\prime}\right]$, however, $\varphi([Z(G)]) \neq[Z(H)]$, and $\varphi$ maps some abelian subgroups of $G$ onto non-abelian subgroups of $H$.

2. The ordinary case. We now return to the proof of the Theorem. For this, the following characterization will be useful.

Lemma 2.1. (See [5, Theorem 3.2]). Every metacyclic p-group $G$ has a presentation $G=\left\langle a, b \mid a^{p^{m}}=1, b^{p^{n}}=a^{p^{m-s}}, a^{b}=a^{\varepsilon+p^{m-c}}\right\rangle$, where $\varepsilon= \pm 1$. Let $r=\varepsilon+p^{m-c}$. The following types arise:

Ordinary metacyclic groups. $p \geq 3$ or $p=2,4 \mid r-1$ and $c<m-1$ if $m \geq 2$.

(i) Split: $0=s \leq c<\min \{n+1, m\}$;

(ii) Non-split: $\max \{1, m-n+1\} \leq s<\min \{c, m-c+1\}$. 
Exceptional metacyclic groups. $p=2,4 \mid r+1$ and $c<m-1$.

(i) Split: $0=s \leq c<\min \{n+1, m-1\}$;

(ii) Non-split: $1=s, \max \{1, m-n+1\} \leq c<\min \{n, m-1\}$, or $1=s, c=0,1=$ $n<m$ (generalized quaternion).

Throughout this section, we assume that both $G$ and $H$ are metacyclic $p$-groups of ordinary type. As, in particular, all metacyclic $p$-groups of odd order are ordinary, our theorem will follow from the result given here in the case where $p \neq 2$. and $H$.

We first collect some information on the parameters related to the presentations of $G$

LeMma 2.2. If $\mathscr{C}(G)$ and $\mathscr{C}(H)$ are order-isomorphic then

(a) $m+n=m^{\prime}+n^{\prime}$,

(b) $\max \{m, n+s\}=\max \left\{m^{\prime}, n^{\prime}+s^{\prime}\right\}$,

(c) $\{m-c, n\}=\left\{m^{\prime}-c^{\prime}, n^{\prime}\right\}$,

(d) $c=c^{\prime}$.

Proof. By $\left[5\right.$, p. 119], we have $|G|=p^{m+n}, \exp (G)=p^{\max \{n+s . m\}}, G / G^{\prime} \cong \mathbb{Z}_{p^{m} \cdots \times \mathbb{Z}_{p^{n}}}$ and $G^{\prime}=\left\langle a^{\rho^{\prime \prime-r}}\right\rangle \cong \mathbb{Z}_{p}$. By Proposition 1.2 and $[1$, Theorem $\mathrm{A}]$, the result follows.

Proposition 2.3. Let $\mathscr{C}(G)$ and $\mathscr{C}(H)$ be order-isomorphic and assume that both $G$ and $H$ are of ordinary type. Then $G \cong H$.

Proof. According to $[5$, p. 110], we have to distinguish the cases when $G$ (resp. $H$ ) is split or non-split, respectively. For simplicity, we shall only consider the case where $G$ is split $(s=0)$ and $H$ is non-split $\left(s^{\prime} \geq 1\right)$, the other ones being similar (see also [5, p. 130]).

In addition to the information given in Lemma 2.1, we also have:

$$
\max \left\{1, m^{\prime}-n^{\prime}+1\right\} \leq s^{\prime}<\min \left\{c, m^{\prime}-c+1\right\} .
$$

By Lemma 2.2(c), we either have $n=n^{\prime}$ or $n=m^{\prime}-c$.

Case 1. $n=n^{\prime}$. Then 2.2(a) implies $m=m^{\prime}$ and 2.2(b) yields $\max \{m, n\}=\max \{m, n+$ $\left.s^{\prime}\right\}$. If $n \geq m$, then this implies $n=\max \{m, n\}=n+s^{\prime}$, so $s^{\prime}=0$, a contradiction. If $n<m$, then $m=\max \left\{m, n+s^{\prime}\right\}$ and so $n \geq n+s^{\prime}$. Using $\left(^{*}\right)$, this yields $\max \{1, m-n+$ $1\} \leq s^{\prime}$, so $m-n+1 \leq s^{\prime}$ and $m+1 \leq n+s^{\prime} \leq m$, a contradiction.

Case 2. $n=m^{\prime}-c$. Then $m-c=n^{\prime}$, so

$$
m^{\prime}=n+c, \quad n^{\prime}=m-c .
$$

Using Lemma 2.2(b), we have $\max \{m, n\}=\max \left\{n+c, m-c+s^{\prime}\right\}$. Case 1 .

(i) If $m \geq n$ and $n+c \geq m-c+s^{\prime}$, then, by $\left({ }^{* *}\right), m=n+c=m^{\prime}$, and we are in

(ii) If $m \geq n$ and $n+c<m-c+s^{\prime}$, then $m=m-c+s^{\prime}$, so $c=s^{\prime}$, but (*) implies $s^{\prime}<c$, a contradiction.

(iii) If $m<n$ and $n+c \geq m-c+s^{\prime}$, then $n=n+c$ and $c=0$. Hence $G$ is abelian. But [1] implies $G \cong H$, a contradiction, since $G$ splits and $H$ does not split (see also [5, p. 110]).

(iv) If $m<n$ and $n+c<m-c+s^{\prime}$, then $n=m-c+s^{\prime}$, so $n+c=m+s^{\prime}$. But $n+c<m-c+s^{\prime} \leq m+s^{\prime}$, a contradiction.

Thus, in this case $\mathscr{C}(G)$ cannot be order-isomorphic to $\mathscr{C}(H)$. 
3. The case $p=2$. In this case let $G=\left\langle a, b \mid a^{2^{m}}=1, b^{2 n}=a^{2^{m-s}}, a^{b}=a^{ \pm 1+2^{m-r}}\right\rangle$ be a metacyclic 2-group and set $A=\langle a\rangle$. The following criterion shows that it can be read off from the poset $\mathscr{C}(G)$ whether or not $Z(G)$ is cyclic:

Lemma 3.1. Let $G$ be a metacyclic 2-group and let $V \leq G$ be a Klein group. The following are equivalent:

(i) $V \leq Z(G)$.

(ii) $[[1],[V]]$ is a modular lattice with three atoms.

Proof. Clearly (i) implies (ii). For the converse, assume by way of contradiction that $V \neq Z(G)$. As $G / A$ is cyclic we must have $V \cap A \neq 1$, so that $V \cap Z(G) \neq 1$. Let $V \cap Z(G)=\langle z\rangle$ and let $v \in V \backslash\langle z\rangle$ be an involution.

If $\Omega_{1}(G) \leq V$ then clearly $v \in Z(G)$ and $V \leq Z(G)$, a contradiction. Hence there exists an involution $u \in G \backslash V$. Let $D=\langle u, v\rangle$, so $D$ is a dihedral group. As above, we have $D \cap A \neq 1$, so that $z \in D$. This implies $V=\langle z, v\rangle \leq D$. Moreover, $v \in D \backslash V$, so that $|D| \geq 8$. By the structure of $D$, we see that $v$ and $v z$ are conjugate in $D$, hence in $G$, but this contradicts (ii).

Corollary 3.2. Let $G$ and $H$ be metacyclic 2-groups and assume $\mathscr{C}(G) \cong \mathscr{C}(H)$. If $Z(G)$ is noncyclic then $Z(H)$ is noncyclic.

It may be noted that two most natural generalizations of Lemma 3.1 are not true. Indeed, part (a) of the following example shows that the hypothesis of $G$ being metacyclic cannot be dispensed with. Moreover, $\mathscr{C}(G)$, in general, is not a lattice, even for $p$-groups. Part (b) shows that $V$, in general, cannot be replaced by an arbitrary noncyclic, abelian group.

ExAmple 3.3. (a) Let $G=\mathbb{Z}_{2}$ wr $\mathbb{Z}_{4}$. Then $G$ is a split extension of an elementary abelian 2-group $B$ of order 16 by a group $C=\langle g\rangle$ of order 4 . Let $A=\left\{\left(x_{1}, \ldots, x_{4}\right) \in\right.$ $\left.B \mid x_{1}+\cdots+x_{4}=0\right\}$. Let $u=(1,0,0,0), v=(1,1,1,0)$ and $w=v^{g}=(0,1,1,1)$. We claim that the Klein groups $K_{1}=\langle u, v\rangle$ and $K_{2}=\langle u, w\rangle$ are not conjugate in $G$. Thus $\left[K_{1}\right]$ and $\left[K_{2}\right]$ are distinct least upper bounds for $[\langle u\rangle]$ and $[\langle v\rangle]=\left[\left\langle v^{g}\right\rangle\right]$ in $\mathscr{C}(G)$ and hence $\mathscr{C}(G)$ is not a lattice. Indeed, assume $K_{2}=K_{1}^{x}$ for some $x \in G$. If $u^{x}=u$ then $x \in C_{G}(u)=B$ and $K_{1}=K_{2}$, a contradiction. If $u^{x}=u w$ then $w=[u, x] \in[B, G]=A$, another contradiction. If $u^{x}=w=v^{g}$ then $u$ and $v$ would be conjugate, a contradiction. So $\mathscr{C}(G)$ is not a lattice. Finally, $K_{1} \neq Z(G)$, but its three subgroups of order 2 are not conjugate in $G$.

(b) Let $G=\left\langle a, b \mid a^{9}=b^{9}=1, a^{b}=a^{4}\right\rangle$ and let $S=\left\langle a, b^{3}\right\rangle$. Then $S \cong \mathbb{Z}_{3} \times \mathbb{Z}_{9}$ and the interval [[1], [S]] of $\mathscr{C}(G)$ is order-isomorphic to the lattice of all subgroups of $S$. But $S \neq Z(G)$.

Proposition 3.4. Let $G$ and $H$ be metacyclic 2-groups and let $\varphi: \mathscr{C}(G) \rightarrow \mathscr{C}(H)$ be an order-isomorphism. Let $N \triangleleft G$ and let $\varphi([N])=[R]$. Then $R \triangleleft H$.

Proof. By [1], the result is true if $G$ is abelian. Moreover, by Proposition 1.2, we have $\varphi\left(\left[G^{\prime}\right]\right)=\left[H^{\prime}\right]$. We proceed by induction on $|G|$.

First, we prove the result in the case $|N|=2$. Here we have $N \leq Z(G)$. If $Z(G)$ is cyclic then $N$ is its unique subgroup of order 2 and we have $N \leq G^{\prime}$, because $G^{\prime}$ is cyclic. 
By the above remark, we have $[R]=\varphi([N]) \leq \varphi\left(\left[G^{\prime}\right]\right)=\left[H^{\prime}\right]$, and so $R \leq H^{\prime}$, which is cyclic, and therefore $R \triangleleft H$, as desired. If $Z(G)$ is noncyclic, then Corollary 3.2 implies that $Z(H)$ is noncyclic. As $H$ is metacyclic, $Z(H)$ contains every element of order 2 , so that $R \leq Z(H)$ and $R \triangleleft H$.

In the general case, let $1 \neq K \triangleleft G$ and choose $N \triangleleft G,|N|=2$, such that $N \leq K$. By the above, if $[R]=\varphi([N])$, then $R \triangleleft H$ and there exists an order-isomorphism $\bar{\varphi}: \mathscr{C}(G / N) \rightarrow \mathscr{C}(H / R)$ which, by induction, maps $[K / N]$ onto the class consisting of a normal subgroup $L / R$ of $H / R$. Thus $\varphi([K])=[L]$ and $L \triangleleft H$.

Remark. An analogous argument to the one used above, in conjunction with our main theorem, proves that Lemma 3.4 holds for every prime $p$.

We now characterize exceptional 2-groups, so let $G$ be of type - and let $H$ be an exceptional 2-group with parameters $m^{\prime}, n^{\prime}, s^{\prime}, c^{\prime},-$. We now show that $m-c$ can be read off from $\mathscr{C}(G)$.

Lemma 3.5. Let $G$ and $H$ be exceptional 2-groups and assume that $\mathscr{C}(G)$ and $\mathscr{C}(H)$ are order-isomorphic. Then $m-c=m^{\prime}-c^{\prime}$.

Proof. Let $\varphi$ be an isomorphism from $\mathscr{C}(G)$ to $\mathscr{C}(H)$. Let $G_{D}$ be the intersection of all normal subgroups $X$ of $G$ such that $G / X$ is a dihedral group. Define $H_{D}$ similarly. By [5, Theorem 4.9], either $G$ is isomorphic to the generalized quaternion group of order $2^{m+1}$ or $G / G_{D}$ is a dihedral group of order $2^{m-c+1}$. In the first case, by [1, Corollary 2] we have $G \cong H$. In the second case, let $\varphi\left(\left[G_{D}\right]\right)=[R]$. Then Proposition 3.4 implies that $R \triangleleft H$ and clearly $\mathscr{C}\left(G / G_{D}\right)$ and $\mathscr{C}(H / R)$ are order-isomorphic. Thus [1, Corollary 2] implies that $H / R$ is a dihedral group, so that $H_{D} \leq R$. An analogous argument applies to $\varphi^{-1}$ and we arrive at $H_{D}=R$. The result follows from the aforementioned result of King.

The following shows that we need to consider the splitting type of exceptional 2-groups.

Lemma 3.6. Let $G$ and $H$ be exceptional metacyclic 2-groups and assume that $\mathscr{C}(G)$ and $\mathscr{C}(H)$ are order-isomorphic. Then $m=m^{\prime}, n=n^{\prime}$ and $c=c^{\prime}$.

Proof. By [5, Proposition 4.10], we have $m+n=m^{\prime}+n^{\prime}$ and $\max \{n+s, m\}=$ $\max \left\{n^{\prime}+s^{\prime}, m^{\prime}\right\}$. Moreover, [5, p. 120] implies $G / G^{\prime} \cong \mathbb{Z}_{2^{\prime \prime}} \times \mathbb{Z}_{2}, H / H^{\prime} \cong \mathbb{Z}_{2^{\prime \prime}} \times \mathbb{Z}_{2}$, and so $n=n^{\prime}$. It follows that $m=m^{\prime}$. Finally, by Lemma 3.5 we have $m-c=m^{\prime}-c^{\prime}$, hence $c=c^{\prime}$.

Lemma 3.7. Let $G$ and $H$ be exceptional metacyclic 2-groups and assume that $\mathscr{C}(G)$ and $\mathscr{C}(H)$ are order-isomorphic. If $G$ splits, also $H$ splits.

Proof. By [5, p. 110], a metacyclic group either splits or never splits. Suppose that $G$ splits. Then $\langle a\rangle \cap\langle b\rangle=1$. Let $\varphi: \mathscr{C}(G) \rightarrow \mathscr{C}(H)$ be the order-isomorphism and let $[R]=\varphi([\langle a\rangle]),[S]=\varphi([\langle b\rangle])$. Then $R$ and $S$ are cyclic, by [1, Proposition 3] and Proposition 3.4 implies $R \triangleleft H$. Moreover, $[1]=[\langle a\rangle \cap\langle b\rangle]$ is the greatest lower bound of $|\langle a\rangle|$ and $[\langle b\rangle]$ in $\mathscr{C}(G)$, so $R \cap S=1$. Finally, we have $|R|=|\langle a\rangle|,|S|=|\langle b\rangle|$ and $|H|=|G|$, hence $H=R S$ is a split extension of $R$ by $S$, which means that $H$ splits.

Proposition 3.8. Let $G$ and $H$ be metacyclic 2-groups of type -, and assume that $\mathscr{C}(G)$ and $\mathscr{C}(H)$ are order-isomorphic. Then $G \cong H$. 
Proof. By Lemma 3.6 we have $m=m^{\prime}, n=n^{\prime}$ and $c=c^{\prime}$. Lemma 3.7 implies $s=s^{\prime}=0$ or $s, s^{\prime} \neq 0$, but in this case by Lemma 2.1 we must have $s=s^{\prime}=1$. The conclusion now holds again by Lemma 2.1 .

Finally, we deal with the case where $G$ is an ordinary and $H$ an exceptional 2-group. In fact, we prove a slightly more general result.

Proposition 3.9. Let $G$ and $H$ be metacyclic 2-groups and assume that $G$ is of type + and $H$ of type -. Then $\mathscr{C}(G)$ is not order-isomorphic to $\mathscr{C}(H)$.

Proof. By Lemma 2.1, $G$ is not isomorphic to $H$. By [5, p. 120], we have $G / G^{\prime} \cong \mathbb{Z}_{2^{m-c}} \times \mathbb{Z}_{2^{n}}$ and $H / H^{\prime} \cong \mathbb{Z}_{2^{n^{\prime}}} \times \mathbb{Z}_{2}$. So $G / G^{\prime} \cong H / H$; implies $\{m-c, n\}=\left\{n^{\prime}, 1\right\}$. If $m=1$, then $G$ would be abelian and so $G \cong H$ by [1], a contradiction. So assume $m \geq 2$. If $m-c=1$ then $c=m-1$, but this contradicts [5, p. 110]. Hence $n=1$ and $G$ has a cyclic normal subgroup of index 2 . Again by $G / G^{\prime} \cong H / H^{\prime}$, an analogous property holds for $H$. Now, [4, p. 91] implies that $H$ (of type - ) is of maximal class. But then by [1, Corollary 2] we have $G \cong H$, a contradiction, because $G$ is of type + .

This also completes the proof of our main theorem.

Acknowledgement. The first named author wishes to express his sincerest thanks for the warm and excellent hospitality offerered by Marusca, Riccardo and "Nonna Gigliola" and for the financial support given by the Dipartimento di Matematica dell'Università di Bologna, during the time of writing this paper.

\section{REFERENCES}

1. R. Brandl, Posets of subgroups in p-groups. Comm. Algebra, 20 (10) (1992), 3043-3054.

2. R. Brandl, A. Caranti and C. M. Scoppola, Metabelian thin p-groups, Quart. J. Math. (Oxford), (2) 43 (1992), 157-173.

3. H. Heineken, Charakterisierung von Gruppen durch gewisse Untergruppenverbände, J. Reine Angew. Math. 220 (1965), 30-36.

4. B. Huppert, Endliche Gruppen I, Springer-Verlag, Berlin Heidelberg New York 1967. 103-131

5. B. W. King, Presentations of metacyclic groups. Bull. Austral. Math. Soc. 8 (1973),

Mathematisches InSTITUT

Am HUBland 12

D-W-8700 WÜRZBURG

GERMANY
Dipartimento di Matematica

Piazza di Porta San Donato, 5

I-40127 BOLOGNA

ITALY 